\title{
Biomarker for recurrent immunoglobulin A nephropathy in kidney allografts: promising but still a long way to go
}

\author{
Byung Ha Chung \\ Department of Internal Medicine, College of Medicine, The Catholic University of Korea, Seoul, Republic of Korea
}

\section{See Article on Page 317-324}

Immunoglobulin A (IgA) nephropathy (IgAN), the most common primary glomerulonephritis worldwide, frequently leads to end-stage renal disease, which requires renal replacement therapy, including kidney transplantation (KT). Recurrence of IgAN after KT was reported to range from $35 \%$ to $60 \%$, with a graft loss rate of $7 \%-10 \%$ [1]. Diagnosis of recurrent IgAN is dependent upon a pathologic diagnosis by an invasive allograft biopsy, and no effective noninvasive methods have been successfully proposed. In this regard, the KDIGO guidelines for care of KT recipients recommend screening for IgAN recurrence using urinalysis and kidney function tests [2]. KT recipients await serological biomarkers for a noninvasive determination of recurrence or severity of IgAN in kidney allograft. To discuss the possibility of biomarkers for recurrent IgAN in kidney allograft, we need to understand the proposed mechanism of development of IgAN. IgAN is characterized by IgA deposits in the kidney, resulting in mesangial cell proliferation, extracellular matrix expansion, and inflammation, finally progressing to fibrosis. Although the origin of the disease is under investigation, aberrantly glycosylated IgAl might be a key element in the pathogenesis of the disease [3]. Defective galactosylation can lead to self-polymerization of IgAl and facilitate its deposition in the kidney. An autoimmune response against the galactose-deficient (Gd) IgAl molecule is initiated, with production of glycan-specific immunoglobulin G (IgG) or IgA autoantibodies. Therefore, IgAN can be caused by immune complex deposition resulting from generation of IgG and IgA antibodies to aberrant nonglycosylated IgAl [3]. Based on this information, serum levels of "Gd-IgAl," "Gd-IgAl-specific IgG and IgA," and "its related immune complexes" have been proposed as biomarkers for progression of IgAN in the native kidney [4]. A study by Berthoux et al. [5] analyzed serum samples from 97 patients with IgAN, 30 healthy volunteers, and 30 patients with non-IgAN disease. The study found that the level of the putative antigen anti-glycosylated IgAl correlated with the disease process in the native kidney.

It has been suggested that this hypothesis for pathogenesis of IgAN can be adapted to an allograft [6]. Therefore, these serological biomarkers might be suitable for characterization of the stage of recurrent IgAN in kidney allografts. To date, four reports (including the current study [7]) investigated the role of serum Gd-IgAl or its related autoantibody/immune complexes for prediction of recurrent IgAN in a kidney allograft (Table 1). Berthelot et al. [8] reported the predictive

Received: May 11, 2021; Revised: May 12, 2021; Accepted: May 12, 2021

Editor: Tae-Hyun Yoo, Yonsei University, Seoul, Republic of Korea

Correspondence: Byung Ha Chung

Department of Internal Medicine, College of Medicine, The Catholic University of Korea, 222 Banpo-daero, Seocho-gu, Seoul 06591, Republic of Korea. E-mail: chungbh@catholic.ac.kr 
Table 1. Published reports of serologic biomarkers for recurrent lgAN after KT

\begin{tabular}{|c|c|c|c|c|c|c|}
\hline Study & Year & Patient group & Measured biomarker & Sampling time & $\begin{array}{l}\text { Observation } \\
\text { period (yr) }\end{array}$ & Result \\
\hline \multirow[t]{3}{*}{ Berthelot et al. [8] } & 2015 & 38 KTRs (recurrence) & Serum Gd-lgA1 & At transplant & $8.7 \pm 2.5$ & $\begin{array}{l}\text { All three markers predicted } \\
\text { recurrence of IgAN }\end{array}$ \\
\hline & & 22 KTRs (non-recurrence) & $\begin{array}{l}\text { IgG anti-lgA } \\
\text { autoantibodies }\end{array}$ & & & \\
\hline & & $17 \mathrm{HCs}$ & $\begin{array}{l}\text { IgA-soluble CD89 } \\
\text { complexes }\end{array}$ & & & \\
\hline \multirow[t]{2}{*}{ Berthoux et al. [9] } & 2017 & 96 KTRs & Serum Gd-lgA1 & $\begin{array}{l}\text { At diagnosis of IgAN } \\
\text { (pretransplant), }\end{array}$ & $12.4 \pm 6.1$ & $\begin{array}{l}\text { Only IgG predicted clinic- } \\
\text { pathologic recurrence of } \\
\text { IgAN }\end{array}$ \\
\hline & & $30 \mathrm{HCs}$ & $\begin{array}{l}\text { IgA1-specific IgG and } \\
\text { IgA autoantibody }\end{array}$ & At transplant & & \\
\hline \multirow[t]{4}{*}{ Temurhan et al. [10] } & 2017 & 18 KTRs (recurrence) & Serum Gd-lgA1 & Posttransplant & $7.0 \pm 3.0$ & $\begin{array}{l}\text { Serum Gd-lgA1 predicted } \\
\text { recurrence of IgAN }\end{array}$ \\
\hline & & 23 KTRs (non-recurrence) & & & & \\
\hline & & 44 non-KT IgAN patients & & & & \\
\hline & & $11 \mathrm{HCs}$ & & & & \\
\hline \multirow[t]{2}{*}{ Park et al. [7] } & 2021 & 14 KTRs (recurrence) & Serum Gd-lgA1 & Posttransplant & $12.8 \pm 7.0$ & $\begin{array}{l}\text { Serum Gd-lgA1 predicted } \\
\text { recurrence of lgAN }\end{array}$ \\
\hline & & 13 KTRs (non-recurrence) & & & & \\
\hline
\end{tabular}

Gd-lgA1, galactose-deficient immunoglobulin A1; HC, healthy controls; IgAN, immunoglobulin A nephropathy; IgG, immunoglobulin G; KT, kidney transplant; KTR, KT recipient.

value of Gd-IgAl for IgAN recurrence in allograft kidneys for the first time. They analyzed three markers, Gd-IgA1, IgG anti-IgA autoantibodies, and IgA-soluble CD89 complexes, using serum obtained at pretransplant in $38 \mathrm{KT}$ recipients, and their results showed that all three markers significantly predicted disease recurrence. In another study by Berthoux et al. [9] the prognostic significance of the levels of Gd-IgAl autoantigen and Gd-IgAl-specific IgG and IgA autoantibodies in serum obtained at the time of transplant or native-kidney IgAN diagnosis was assessed for clinicopathologic recurrence, allograft failure, and patient death over 10 years. Compared to healthy controls, the patients had significantly elevated serum Gd-IgAl level at diagnosis and transplant, but the level was not associated with any outcomes, including IgAN recurrence. In contrast, the level of serum Gd-IgA1specific IgG autoantibodies at transplant was associated with a higher risk of recurrence. In contrast to previous studies that used samples at pretransplant or at transplant, a more recent study [10] measured serum Gd-IgAl level at a mean time of $51 \pm 29$ months after KT. As a result, the level of GdIgG1 in recurrent IgAN patients was significantly higher than those in nonrecurrent IgAN patients or healthy controls. In a current study, Park et al. [7] enrolled $27 \mathrm{KT}$ recipients who underwent allograft biopsy and measured the serum Gd-
IgA1 level using serum collected at the allograft biopsy. The mean serum Gd-IgAl level was significantly higher in the recurrent IgAN group than in the nonrecurrent IgAN group, and serum Gd-IgA1 level was an independent factor predicting IgAN recurrence. They concluded that serum Gd-IgA1 could be used as a diagnostic biomarker for recurrent IgAN in KT.

All the above studies found that serum Gd-IgAl or its related autoantibody level showed significance in predicting recurrent IgAN in kidney allograft. However, many obstacles must be overcome or clarified before these biomarkers can be applied in clinical practice. First, the previous studies used Gd-IgAl level at specific time points including pretransplant, at KT, or at allograft biopsy. Thus, the dynamics of Gd-IgAl level during the posttransplant period and their association to IgAN recurrence after KT were not shown. Therefore, it is unclear whether Gd-IgAl level remained high and induced IgAN recurrence, or whether it was low but became high at the time of recurrence. To clarify this issue, longitudinal assessment in a prospective cohort should determine the prototypical course of Gd-IgAl level and its association with clinicopathologic recurrence of IgAN in allograft. Second, most studies did not show an association between Gd-IgAl level and clinical outcomes, such as al- 
lograft failure, even though it was useful in predicting IgAN recurrence. Perhaps an association was not demonstrated because previous studies were performed in a relatively small-sized patient group. If there is no correlation with allograft survival, the need for biomarkers will be halved. Furthermore, an effective therapeutic strategy for recurrent IgAN has not been established. Hence, strategies to improve allograft outcomes when recurrent IgAN is suspected based on high Gd-IgAl level remain unclear. All KT patients receive strong maintenance immunosuppression, so few other treatments can be added in recurrent IgAN. Hence, new therapeutic agents to change the clinical outcomes need to be developed to maximize the effectiveness of biomarkers for recurrent IgAN. Lastly, the assay method needs to be standardized. In earlier studies, serum Gd-IgAl level was measured by lectin enzyme-linked immunosorbent assay using Helix aspersa agglutinin, a lectin that binds to terminal galactosyl- $N$-acetylamine residues $[7,8]$. In contrast, the current study [7] and the study by Temurhan et al. [10] used a lectin-independent Gd-IgAl assay (Immuno-Biological Laboratories Co., Ltd., Gunma, Japan). In addition, the significant level associated with IgAN recurrence was different between the studies. Therefore, standardization of measurement methods to overcome inter- or intralaboratory disparity should be achieved.

In summary, theoretically, serum Gd-IgA level has potential as a biomarker for recurrent IgA nephropathy. Areas that require further investigation, however, include an understanding of IgAN recurrence, especially the dynamics during the posttransplant period, and the effectiveness of serum Gd-IgA level for predicting allograft survival. A prospective multicenter study including serial protocol biopsies and measurement of serum Gd-IgAl level by nephrologists involved in KT should further clarify these issues.

\section{Conflicts of interest}

The author has no conflicts of interest to declare.

\section{ORCID}

Byung Ha Chung, https://orcid.org/0000-0003-0048-5717

\section{References}

1. Graves RC, Fine RN. Kidney retransplantation in children following rejection and recurrent disease. Pediatr Nephrol 2016;31:2235-2247.

2. Kidney Disease: Improving Global Outcomes (KDIGO) Transplant Work Group. KDIGO clinical practice guideline for the care of kidney transplant recipients. Am J Transplant 2009;9 Suppl 3:S1-S155.

3. Wyatt RJ, Julian BA. IgA nephropathy. N Engl J Med 2013; 368:2402-2414.

4. Suzuki H. Biomarkers for IgA nephropathy on the basis of multihit pathogenesis. Clin Exp Nephrol 2019;23:26-31.

5. Berthoux F, Suzuki H, Thibaudin L, et al. Autoantibodies targeting galactose-deficient IgA1 associate with progression of IgA nephropathy. J Am Soc Nephrol 2012;23:1579-1587.

6. Sofue T, Suzuki H, Ueda N, Kushida Y, Minamino T. Post-transplant immunoglobulin A deposition and nephropathy in allografts. Nephrology (Carlton) 2018;23 Suppl 2:4-9.

7. Park WY, Kim Y, Paek JH, Jin K, Han S. Clinical significance of serum galactose-deficient immunoglobulin A1 for detection of recurrent immunoglobulin A nephropathy in kidney transplant recipients. Kidney Res Clin Pract 2021;40:317-324.

8. Berthelot L, Robert T, Vuiblet V, et al. Recurrent IgA nephropathy is predicted by altered glycosylated IgA, autoantibodies and soluble CD89 complexes. Kidney Int 2015;88:815-822.

9. Berthoux F, Suzuki H, Mohey H, et al. Prognostic value of serum biomarkers of autoimmunity for recurrence of IgA nephropathy after kidney transplantation. J Am Soc Nephrol 2017;28:19431950.

10. Temurhan S, Akgul SU, Caliskan Y, et al. A novel biomarker for post-transplant recurrent IgA Nephropathy. Transplant Proc 2017;49:541-545. 